\title{
Adaptive Control with ESP32 for Anti-windup Design of Actuator with Input Saturation
}

\author{
Fei $\mathrm{Xu}^{1, \mathrm{a}^{*}}$, Liping Wang ${ }^{1, \mathrm{~b}}$ and Rongrong Wang ${ }^{1, \mathrm{c}}$ \\ ${ }^{1}$ Xijing University, Xi'an, Shaanxi, China \\ axofun@qq.com; bwangliping@xijing.edu.cn; ‘381713963@qq.com \\ *The corresponding author
}

\begin{abstract}
Keywords: Electro-hydrostatic actuator; Servo system; Adaptive backstepping control; Antiwindup control
\end{abstract}

\begin{abstract}
For overcoming the unknown nonlinearity and parameters variability in the model of electro-hydrostatic actuator system, A Lyapunov function-based control algorithm is addressed for force tracking control in the case of input saturation presence. Treating modeling errors, unmodeled nonlinearities as unknown external disturbances, the state space model of electro-hydrostatic actuator is formulated as a strict feedback system. By using backstepping approaches, an online estimation scheme is adopted to approximate the external disturbance of the system. To deal with saturation, the anti-windup compensator is constructed. The tracking error is adjustable by an explicit choice of design parameters. The simulation results and experiment results are provided to show that the control scheme proposed has a good capacity to deal with the model parameter uncertainty, and compared with the classical beckstepping control, the robustness and steady state accuracy have improved obviously.
\end{abstract}

\section{Introduction}

The rapid development of aviation makes the defects of traditional hydraulic actuation mechanism get serious. With the rapid development of the aviation field, the shortcomings of the traditional hydraulic actuator are more and more prominent. In recent years, with the development of power telex technology, especially the use of high-voltage DC power system, the electromechanical actuator (EMA) in aircraft applications become a reality.

Electro-Hydrostatic Actuator (EHA) has the advantages of hydraulic system and motor actuation, can be used as a backup of traditional hydraulic actuator [2] constitute a redundant system, or with the EMA constitute a hybrid actuation system [3], has good prospects for development, has been used in large passenger aircraft A380. Domestic research started late, in the modeling and simulation and prototype verification stage.

In this paper, the difference between the controller output and the actual input of the controlled object is used as the feedback signal, and the Anti-windup compensator is designed to form the feedback loop to eliminate the Windup phenomenon. In this paper, the Nussbaum function is used to approximate the differential term of the saturated fitting function. The advantages of the design are simple and easy to realize. Simulation and experiment verify the validity of the control method.

\section{Electric Servo System Structure}

For PMSM of an electric servo sytem, it is assumed that is the magnetic circuit not saturated, the magnetic field of the air gap is sinusoidal, the stator is a three-phase symmetrical winding, the rotor has no damper winding, therefore, the hidden pole PMSM is modeled like this:

The dynamic equation of the load part is:

$$
J_{1} \ddot{\theta}_{1}+b_{1} \dot{\theta}_{1}=\eta T_{1}+T_{\mathrm{f}}
$$

where, $J_{1}$ is the load moment of inertia, $\theta_{1}$ is the load output corner, $b_{1}$ is the load viscidity damping coefficient, $\eta$ is the transmission mechanism deceleration ratio, $T_{\mathrm{f}}$ is the disturbance torque. 
Due to the backlash nonlinearity, the relationship between the load torque $T_{1}$ and the relative displacement of the drive mechanism can be represented by the following dead zone model:

$$
T_{1}=\left\{\begin{array}{cc}
k(\Delta \theta-\alpha) & \Delta \theta>\alpha \\
0 & |\Delta \theta| \leq \alpha \\
k(\Delta \theta+\alpha) & \Delta \theta<-\alpha
\end{array}\right.
$$

Where $\Delta \theta=\theta-\eta \theta_{1}, 2 \alpha$ is the backlash width, $k$ is the main driven gear meshing stiffness coefficient. Because the discontinuity of backlash make it difficult to design the controller, therefore, a continuously differentiable function is used to fit the deadband function:

$$
T_{\mathrm{s}}=k \Delta \theta+4 k \alpha \frac{e^{-\gamma \Delta \theta}-1}{e^{-\gamma \Delta \theta}+1}
$$

Where $\gamma$ is a constant Define the fitting error as:

$$
T_{\Delta}=T_{1}-T_{\mathrm{s}}=T_{1}-k \Delta \theta-4 k \alpha \varepsilon(\Delta \theta)
$$

Where $\varepsilon(\Delta \theta)=\left(e^{-\gamma \Delta \theta}-1\right) /\left(e^{-\gamma \Delta \theta}+1\right)$

From (4) we obtain:

$$
T_{1}=k\left(\theta-\eta \theta_{1}\right)+4 k \alpha \varepsilon\left(\theta-\eta \theta_{1}\right)+T_{\Delta}
$$

Substituting equation (5) into equation (1), the mechanical equation of PMSM can be written as:

$$
\begin{aligned}
& \frac{d \omega}{d t}=\frac{3 p \varphi_{f}}{2 J} i_{q}-\frac{b_{\mathrm{m}}}{J} \omega-\frac{T_{1}}{J} \\
& =\frac{\eta k}{J} \theta_{1}-\frac{k}{J} \theta-\frac{b_{\mathrm{m}}}{J} \omega+\frac{3 p \varphi_{f}}{2 J} i_{q}-\frac{4 k \alpha \varepsilon\left(\theta-\eta \theta_{1}\right)}{J}+d_{2}(t) \\
& d_{2}(t)=-\frac{T_{\Delta}}{J}
\end{aligned}
$$

Control objectives: the equation (6) described by the design of the servo-servo model adaptive controller to achieve the desired corner signal $\theta_{1}^{*}(t)$ tracking, closed-loop system to achieve asymptotic stability, all signals are bounded.

\section{Mathematical Model of EHA Pressure Control}

EHA mathematical model can be calculated by the joint module. for convenence, we only consider the modeling process under the normal working conditions, ignore the one-way valve, throttle valve, safety valve and other hydraulic accessories. For the surface mount brushless DC motor, assuming the magnetic circuit is not saturated, excluding eddy current and hysteresis loss, the voltage and current equation can be written as:

$$
U=R i+L \frac{d i}{d t}+E_{\mathrm{a}}
$$

In Equation (1), the armature voltage, the stator resistance, the armature current, the stator inductance, the armature back-EMF, can be expressed as:

$$
E_{\mathrm{a}}=k_{\mathrm{e}} \omega
$$

Where, $k_{\mathrm{e}}$ is the counter electromotive force coefficient, $\omega$ is the electrical machinery rotational speed. Motor and hydraulic pumpcoaxial connected, regardless of friction torque loss, the motor torque applied to the pump:

$$
T=k_{\mathrm{T}} i=\left(J_{\mathrm{m}}+J_{\mathrm{p}}\right) \dot{\omega}+\left(k_{\mathrm{m}}+k_{\mathrm{p}}\right) \omega+D\left(P_{\mathrm{A}}-P_{\mathrm{B}}\right)
$$

In Equation (9), $k_{\mathrm{T}}$ is the torque coefficient, $J_{\mathrm{m}}$ and $J_{\mathrm{p}}$ is the rotational inertia of the motor and 
the pump, $k_{\mathrm{m}}$ and $k_{\mathrm{p}}$ are the viscous damping coefficient of the motor and pump respectively, $D$ is the displacement of the pump, $P_{\mathrm{A}}$ and $P_{\mathrm{B}}$ are the inlet and outlet pressure of the pump.

\section{Stability Analysis}

Three ways are designed to test the algorithm performance: excluding the adaptive backstepping sliding control mode; excluding integral adaptive backstepping sliding mode control mode; excluding constraints of the adaptive backstepping sliding control mode. Marked with algorithm a, b, c respectively in Fig. 1. Here are the differences: let $\rho_{1}=\rho_{2}=0, \gamma_{1}=\gamma_{2}=0$ in algorithm a; let $\lambda=0$ in algorithm $\mathrm{b}$; and algorithm $\mathrm{c}$ is the conventional algorithm, and the rest paremeters remain unchange. The results are shown in Fig. 1:

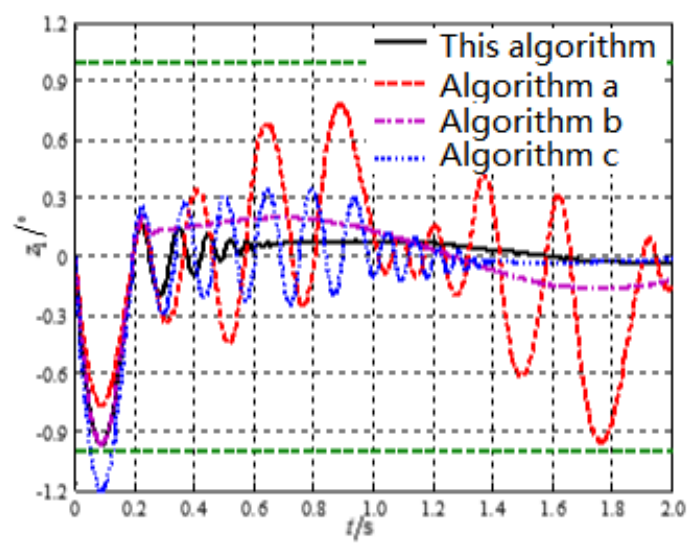

Figure 1. Test results

Compared with the experimental results of algorithm a, it can be seen that parameter adaptive law is very important to increase the system dynamic performance, and its control quality can be improved significantly by compensating the actual system uncertainties while on-line estimation. Compared with the experimental results of algorithm $b$, the design of the integral term in the backstepping process has positive significance for reducing the steady-state error of the system. Compared with the control algorithm c, it can see that the error range is constrained with BLF, thus ensuring the control accuracy. The maximum error and error absolute value integral of actual test are significantly larger than that of simulation. The main reason is that there exists extra torque load simulator. Despite the loading precision is yet to be improved, the strong robustness has been proved by the proposed control method.

\section{Conclusion}

This paper proposes a control input constraints of backlash nonlinearity compensation method, whose convergence of iterative learning control law are stricted proved. Based on the nonlinear model of single servo, the research method is extended to the multi-joint servo system. The deadzone model is approximated by continuously differentiable functions, and the fitting errors and model uncertainties are treated as disturbance-like terms. Therefore,the continuous nonlinear model with uncertain perturbation can realize the accurate compensation of backlash nonlinearity. Compared with the conventional backstepping control, the experimental results show that the control precision and robustness are significantly improved. Theoretical and experimental simulation results have verified the validity and rationality of this method. 


\section{References}

[1] J.M Zheng, S.D Zhao and S.G Wei, Application of self-tuning fuzzy PID controller for a SRM direct drive volume control hydraulic press [J]. Control Engineering Practice, 2009, 17(12): 1398-1404.

[2] B Gao, Yong-ling FU, Zhong-cai PEI, Ji-ming MA. Research on Dual-Variable Integrated Electro-Hydrostatic Actuator [J]. Chinese Journal of Aeronautics, 2006, 19(1): 77-82.

[3] Aeronautics and Astronautics, 2006, 32(05): 553-557.

[4] S.H Cho, R Burton, Position control of high performance hydrostatic actuation system using a simple adaptive control (SAC) method, Mechatronics, 2011, 21(1): 109-115.

[5] Hoang Thinh Do, Hyung Gyu Park, Kyoung Kwan Ahn, Application of an adaptive fuzzy sliding mode controller in velocity control of a secondary controlled hydrostatic transmission system [J]. Mechatronics, 2014(8): 1157-1165.

[6] Hwi-Beom Shin, Jong-Gyu Park.Anti-Windup PID Controller With Integral State Predictor for Variable-Speed Motor Drives [J]. IEEE Transactions on Industrial Electronics, 2012, 59(3):1509-1516.

[7] M Krstic, I Kanellakopoulos, P V Kokotovic. Nonlinear and adaptive control design[M]. New York: Wiley Interscience, 1995: 61-64.

[8] C.Y Wen, J Zhou, ZT Liu, Hongye Su.Robust Adaptive Control of Uncertain Nonlinear Systems in the Presence of Input Saturation and External Disturbance[J]. IEEE Transactions on Automatic Control, 2011, 56(7):1672-1678.

[9] R.J Kang, Z.X Jiao and S.P Wang, et al. Design and simulation of electro-hydrostatic actuator with a built-in power regulator [J]. Chinese Journal of Aeronautics, 2009, 22(6): 700-706.

[10] Andrew Alleyne, Rui Liu, A simplified approach to force control for electro-hydraulic systems [J]. Control Engineering Practice, 2000, 8(12): 1347-1356.

[11]J Zhou, C.Y Wen, Adaptive Backstepping Control of Uncertain Systems - Nonsmooth Nonlinearities, Interactions or Time-Variations [M]. Berlin: Springer, 2008: 189-198. 